\title{
RESEARCH IN THE INTERPOLATION REPRESENTATIONS OF STOCHASTIC PROCESSES IN THE TWO TYPES OF INTERPOLATION KNOTS
}

\author{
Ganna Verovkina \\ Department of Mathematical Physics \\ Taras Shevchenko National University of Kyiv \\ 60 Volodymyrska str., Kyiv, Ukraine, 01033 \\ ganna.verov@gmail.com
}

\begin{abstract}
The article deals with some interpolation representations of stochastic processes with non-equidistance interpolation knots. Research is based on observations of the process and its derivatives of the first and second orders at some types of knots and observations of the process and its derivatives of the first orders at other types of knots. The necessary results from the theory of entire functions of complex variable are formulated. The function bounded on any bounded region of the complex plane is considered. The estimate of the residual of the interpolation series is obtained. The interpolation formula that uses the value of the process and its derivatives at the knots of interpolation is proved. Considering the separability of the process and the convergence of a row that the interpolation row converges to the stochastic process uniformly over in any bounded area of changing of parameter is obtained. The main purpose of this article is the obtained convergence with probability 1 of the corresponding interpolation series to a stochastic process in any bounded domain of changes of parameter. Obtained results may be applied in the modern theory of information transmission.

Keywords: stochastic process, interpolation representation, series, non-equidistance interpolation knots, separability, convergence with probability 1 .

\section{Introduction}

The one of the fundamental results in the Theory of Information Transmission is a theorem of expression of the function with a bounded specter of values in the periodic sequence of initial moments. The significance of that fact was first introduced in [1]. Further these questions were studied in [2, 3]. Kotelnikov-Shannon theorem is generally well-known [4]. In the present time, the investigations related to the construction of interpolation polynoms are attracting significant interest. Many of the questions concerning the construction of a spline approximation are known as representation of a motion in 3D modeling implemented with help of interpolation and approximation [5]. Many questions in modern physics [6] as well as physics of materials [7] and the modern theory of signal transmission [8] are based on the Kotelnikov-Shannon theorem. The questions of interest are constructing interpolation polynoms with non-equidistance interpolation knots. The present work is concerned on the questions stated above.
\end{abstract}

\section{Materials and Methods}

2. 1. Interpolation representation of stochastic processes with the first type of non-equidistance interpolation knots

Let's consider the interpolation representation of stochastic processes [9] on non-equidistance interpolation knots of the type

$$
\begin{gathered}
\mathrm{t}_{\mathrm{n} 0}=\mathrm{n} \frac{5 \pi}{\alpha}, \\
\mathrm{t}_{\mathrm{n} 1}=\mathrm{n} \frac{5 \pi}{\alpha}+\frac{\pi}{\alpha}, \mathrm{n} \in \mathrm{Z}
\end{gathered}
$$

based on observations of the process and its derivatives of the first and second orders at knots $\mathrm{t}_{\mathrm{n} 0}, \mathrm{n} \in \mathrm{Z}$ and observations of the process and its derivatives of the first orders at knots $\mathrm{t}_{\mathrm{n} 1}, \mathrm{n} \in \mathrm{Z}$.

Let's formulate the necessary results from the theory of entire functions of complex variable. 
Lemma 1. Let $\mathrm{f}(\mathrm{z})$ be an entire bounded on the real axis function of exponential type with indicator $\sigma$

Then for any $\alpha, \alpha>\sigma$, the representation holds true

$$
\begin{aligned}
& f(z)=\sum_{n=-\infty}^{\infty}\left(\frac{f\left(t_{n 0}\right)}{\frac{\alpha}{5}\left(z-t_{n 0}\right)} \times \frac{1}{2 \sin ^{2} \frac{\pi}{5}}+\frac{f^{\prime}\left(t_{n 0}\right)}{\left(\frac{\alpha}{5}\left(z-t_{n 0}\right)\right)^{2}} \times \frac{1}{\frac{\alpha}{5} \sin ^{2} \frac{\pi}{5}}+\right. \\
&+\frac{f^{\prime \prime}\left(t_{n 0}\right)}{\frac{\alpha}{5}\left(z-t_{n 0}\right)} \times \frac{1}{2\left(\frac{\alpha}{5} \sin \frac{\pi}{5}\right)^{2}}+\frac{f\left(t_{n 0}+\frac{\pi}{\alpha}\right)}{\left(\frac{\alpha}{5}\left(z-t_{n 0}-\frac{\pi}{\alpha}\right)\right)^{2}} \times \frac{1}{\sin ^{3} \frac{\pi}{5}}+\frac{f^{\prime}\left(t_{n 0}+\frac{\pi}{\alpha}\right)}{\frac{\alpha}{5}\left(z-t_{n 0}-\frac{\pi}{\alpha}\right)} \times \frac{1}{\frac{\alpha}{5} \sin ^{3} \frac{\pi}{5}} \times \\
& \times \sin ^{3} \frac{\alpha}{5}\left(z-t_{n 0}\right) \times \sin ^{2} \frac{\alpha}{5}\left(z-t_{n 0}-\frac{\pi}{\alpha}\right),
\end{aligned}
$$

where $\mathrm{t}_{\mathrm{n} 0}=\mathrm{n} \frac{5 \pi}{\alpha}, \mathrm{n} \in \mathrm{Z}$, provided that the interpolation series (1) converges uniformly in any bounded region of the complex plane.

Proving Lemma 1, as in [10-12], estimation of the residual of the interpolation series is obtained (1), which has the following form

$$
\left|\mathrm{R}_{\mathrm{n}}(\mathrm{z})\right| \leq \mathrm{LG}(\mathrm{z}) \mathrm{C}_{\mathrm{f}} \frac{\alpha}{\alpha-\sigma} \frac{1}{\mathrm{n}}
$$

where $\mathrm{L}$ is a constant,

$$
\begin{gathered}
\mathrm{C}_{\mathrm{f}}=\sup _{\mathrm{t} \in \mathrm{R}}|\mathrm{f}(\mathrm{t})|, \\
\mathrm{G}(\mathrm{z})=\left|\sin ^{3} \frac{\alpha}{5} \mathrm{z} \times \sin ^{2} \frac{\alpha}{5}\left(\mathrm{z}-\frac{\pi}{\alpha}\right)\right|
\end{gathered}
$$

is a function bounded on any bounded region of the complex plane.

Let's consider a stochastic $\xi(t), t \in R$ with $M \xi(t)=0$ and covariance function which representation is

$$
\mathrm{B}(\mathrm{t}, \mathrm{s})=\int_{\Lambda \times \Lambda} \mathrm{f}(\mathrm{t}, \lambda) \overline{\mathrm{f}(\mathrm{s}, \mu)} \mathrm{F}(\mathrm{d} \lambda, \mathrm{d} \mu)
$$

where $\Lambda$ is a set of parameters, $F\left(A_{1}, A_{2}\right)$ is a positive definite additive complex function on $\Lambda \times \Lambda$ such that

$$
\int_{\Lambda \times \Lambda}|\mathrm{F}(\mathrm{d} \lambda, \mathrm{d} \mu)|<+\infty
$$

The function $f(t, \lambda)$ with respect to $t$ is an entire function of exponential type with indicator $\sigma(\lambda)$ such that

$$
\sup _{\lambda \in \Lambda} \sup _{-\infty<t<+\infty}|f(t, \lambda)|=C_{f}<+\infty
$$




$$
\sup _{\lambda \in \Lambda} \sigma(\lambda)=\sigma<+\infty
$$

The following theorem holds true.

Theorem 1. Let $\xi(\mathrm{t})$ be a separable stochastic process that satisfies conditions (3)-(6). Then for any $\alpha, \alpha>\sigma$ with probability 1 the following representation holds true

$$
\begin{aligned}
& \xi(t)=\sum_{n=-\infty}^{\infty}\left(\frac{\xi\left(t_{n 0}\right)}{\frac{\alpha}{5}\left(t-t_{n 0}\right)} \times \frac{1}{2 \sin ^{2} \frac{\pi}{5}}+\frac{\xi^{\prime}\left(t_{n 0}\right)}{\left(\frac{\alpha}{5}\left(t-t_{n 0}\right)\right)^{2}} \times \frac{1}{\frac{\alpha}{5} \sin ^{2} \frac{\pi}{5}}+\right. \\
&\left.+\frac{\xi^{\prime \prime}\left(t_{n 0}\right)}{\frac{\alpha}{5}\left(t-t_{n 0}\right)} \times \frac{1}{2\left(\frac{\alpha}{5} \sin \frac{\pi}{5}\right)^{2}}+\frac{\xi\left(t_{n 0}+\frac{\pi}{\alpha}\right)}{\left(\frac{\alpha}{5}\left(t-t_{n 0}-\frac{\pi}{\alpha}\right)\right)^{2}} \times \frac{1}{\sin ^{3} \frac{\pi}{5}}+\frac{\xi^{\prime}\left(t_{n 0}+\frac{\pi}{\alpha}\right)}{\frac{\alpha}{5}\left(t-t_{n 0}-\frac{\pi}{\alpha}\right)} \times \frac{1}{\frac{\alpha}{5} \sin ^{3} \frac{\pi}{5}}\right) \times \\
& \times \sin ^{3} \frac{\alpha}{5}\left(t-t_{n 0}\right) \times \sin ^{2} \frac{\alpha}{5}\left(t-t_{n 0}-\frac{\pi}{\alpha}\right) .
\end{aligned}
$$

Proof: according to the theorem about spectral representation of stochastic processes [9], we will write the process $\xi(\mathrm{t})$ as follows:

$$
\xi(\mathrm{t})=\int_{\Lambda} \mathrm{f}(\mathrm{t}, \lambda) \mathrm{Z}(\mathrm{d} \lambda)
$$

where $Z(d \lambda)$ is a stochastic measure on $\Lambda$, such that $\operatorname{MZ}\left(A_{1}\right) \cdot \overline{Z\left(A_{2}\right)}=F\left(A_{1}, A_{2}\right)$. For any natural $n$ let's consider a process $\xi_{n}(t)$, which we will define as a partial sum with a number $n$ of row (7).

$$
\begin{aligned}
& \xi_{\mathrm{n}}(\mathrm{t})=\sum_{\mathrm{k}=-\mathrm{n}}^{\mathrm{n}}\left(\frac{\xi\left(\mathrm{t}_{\mathrm{k} 0}\right)}{\frac{\alpha}{5}\left(\mathrm{t}-\mathrm{t}_{\mathrm{k} 0}\right)} \times \frac{1}{2 \sin ^{2} \frac{\pi}{5}}+\frac{\xi^{\prime}\left(\mathrm{t}_{\mathrm{k} 0}\right)}{\left(\frac{\alpha}{5}\left(\mathrm{t}-\mathrm{t}_{\mathrm{k} 0}\right)\right)^{2} \frac{\alpha}{5} \sin ^{2} \frac{\pi}{5}}+\right. \\
&\left.+\frac{\xi^{\prime \prime}\left(\mathrm{t}_{\mathrm{k} 0}\right)}{\frac{\alpha}{5}\left(\mathrm{t}-\mathrm{t}_{\mathrm{k} 0}\right)} \times \frac{1}{2\left(\frac{\alpha}{5} \sin \frac{\pi}{5}\right)^{2}}+\frac{\xi\left(\mathrm{t}_{\mathrm{k} 0}+\frac{\pi}{\alpha}\right)}{\left(\frac{\alpha}{5}\left(\mathrm{t}-\mathrm{t}_{\mathrm{k} 0}-\frac{\pi}{\alpha}\right)\right)^{2}} \times \frac{1}{\sin ^{3} \frac{\pi}{5}}+\frac{\xi^{\prime}\left(\mathrm{t}_{\mathrm{k} 0}+\frac{\pi}{\alpha}\right)}{\frac{\alpha}{5}\left(\mathrm{t}-\mathrm{t}_{\mathrm{k} 0}-\frac{\pi}{\alpha}\right)} \times \frac{1}{\frac{\alpha}{5} \sin ^{3} \frac{\pi}{5}}\right) \times \\
& \times \sin ^{3} \frac{\alpha}{5}\left(\mathrm{t}-\mathrm{t}_{\mathrm{k} 0}\right) \times \sin ^{2} \frac{\alpha}{5}\left(\mathrm{t}-\mathrm{t}_{\mathrm{k} 0}-\frac{\pi}{\alpha}\right) .
\end{aligned}
$$

Using the representation (8) and the statement of the Lemma 1, we will write $\xi_{\mathrm{n}}(\mathrm{t})$ as follows:

$$
\begin{gathered}
\xi_{\mathrm{n}}(\mathrm{t})=\sum_{\mathrm{k}=-\mathrm{n}}^{\mathrm{n}} \int_{\Lambda}\left(\frac{\mathrm{f}\left(\mathrm{t}_{\mathrm{k} 0}\right)}{\frac{\alpha}{5}\left(\mathrm{t}-\mathrm{t}_{\mathrm{k} 0}\right)} \times \frac{1}{2 \sin ^{2} \frac{\pi}{5}}+\frac{\mathrm{f}^{\prime}\left(\mathrm{t}_{\mathrm{k} 0}\right)}{\left(\frac{\alpha}{5}\left(\mathrm{t}-\mathrm{t}_{\mathrm{k} 0}\right)\right)^{2}} \times \frac{1}{\frac{\alpha}{5} \sin ^{2} \frac{\pi}{5}}+\right. \\
\left.+\frac{\mathrm{f}^{\prime \prime}\left(\mathrm{t}_{\mathrm{k} 0}\right)}{\frac{\alpha}{5}\left(\mathrm{t}-\mathrm{t}_{\mathrm{k} 0}\right)} \times \frac{\mathrm{f}\left(\mathrm{t}_{\mathrm{k} 0}+\frac{\pi}{\alpha}\right)}{2\left(\frac{\alpha}{5} \sin \frac{\pi}{5}\right)^{2}}+\frac{\mathrm{f}^{\prime}\left(\mathrm{t}_{\mathrm{k} 0}+\frac{\pi}{\alpha}\right)}{\left(\frac{\alpha}{5}\left(\mathrm{t}-\mathrm{t}_{\mathrm{k} 0}-\frac{\pi}{\alpha}\right)\right)^{2}} \times \frac{1}{\sin ^{3} \frac{\pi}{5}}+\frac{\frac{\alpha}{5}\left(\mathrm{t}-\mathrm{t}_{\mathrm{k} 0}-\frac{\pi}{\alpha}\right)}{\frac{\alpha}{5} \sin ^{3} \frac{\pi}{5}}\right) \times \\
\times \sin ^{3} \frac{\alpha}{5}\left(\mathrm{t}-\mathrm{t}_{\mathrm{k} 0}\right) \times \sin ^{2} \frac{\alpha}{5}\left(\mathrm{t}-\mathrm{t}_{\mathrm{k} 0}-\frac{\pi}{\alpha}\right) \mathrm{Z}(\mathrm{d} \lambda) .
\end{gathered}
$$


Then, based on the representation (1), (8), (9) and the estimation (2), obtain

$$
\mathrm{M} \mid \xi(\mathrm{t}))-\left.\xi_{\mathrm{n}}(\mathrm{t})\right|^{2} \leq \mathrm{R}_{\mathrm{n}}^{2}(\mathrm{t}) \int_{\Lambda \times \Lambda}|\mathrm{F}(\mathrm{d} \lambda, \mathrm{d} \mu)|=\mathrm{L}^{2} \mathrm{G}^{2}(\mathrm{t}) \mathrm{C}_{\mathrm{f}}^{2}\left(\frac{\alpha}{\alpha-\sigma}\right)^{2} \frac{1}{\mathrm{n}^{2}} \int_{\Lambda \times \Lambda}|\mathrm{F}(\mathrm{d} \lambda, \mathrm{d} \mu)| .
$$

From the inequality (10) and considering the condition (4) we obtain the following: an interpolation row (7) converges to $\xi(\mathrm{t})$ in the mean square.

Considering the separability of the process $\xi(t)$ and the convergence of a row

$$
\sum_{n=-\infty}^{\infty}\left|\xi(t)-\xi_{n}(t)\right|^{2}
$$

obtain that the interpolation row (7) converges to the stochastic process $\xi(t)$ almost surely uniformly over $t$ in any bounded area of changing of $t$.

We obtain that the interpolation series (7) converges with probability 1 to a stochastic process $\xi(t)$ in any bounded domain of changes of parameter $t$.

\section{2. Interpolation representation of stochastic processes with the second type of} non-equidistance interpolation knots

Let's consider the interpolation representation of stochastic processes [9] on non-equidistance interpolation knots of the type

$$
\begin{gathered}
\mathrm{t}_{\mathrm{n} 0}=\mathrm{n} \frac{5 \pi}{\alpha}, \\
\mathrm{t}_{\mathrm{n} 1}=\mathrm{n} \frac{5 \pi}{\alpha}+\frac{\pi}{\alpha}, \\
\mathrm{t}_{\mathrm{n} 2}=\mathrm{n} \frac{5 \pi}{\alpha}+\frac{2 \pi}{\alpha}, \mathrm{n} \in \mathrm{Z}
\end{gathered}
$$

based on observations of the process at knots $t_{n 0}, n \in Z$ and observations of the process and its derivatives of the first orders at knots $t_{n 1}, t_{n 2}, n \in Z$.

Let's formulate the necessary results from the theory of entire functions of complex variable.

Lemma 2. Let $\mathrm{f}(\mathrm{z})$ be an entire bounded on the real axis function of exponential type with indicator $\sigma$.

Then for any $\alpha, \alpha>\sigma$, the representation holds true

$$
\begin{aligned}
& \mathrm{f}(\mathrm{z})=\sum_{\mathrm{n}=-\infty}^{\infty}\left(\frac{\mathrm{f}\left(\mathrm{t}_{\mathrm{n} 0}\right)}{\frac{\alpha}{5}\left(\mathrm{z}-\mathrm{t}_{\mathrm{n} 0}\right)} \times \frac{1}{\sin ^{2} \frac{\pi}{5} \sin ^{2} \frac{2 \pi}{5}}+\frac{\mathrm{f}\left(\mathrm{t}_{\mathrm{n} 0}+\frac{\pi}{\alpha}\right)}{\left(\frac{\alpha}{5}\left(\mathrm{z}-\mathrm{t}_{\mathrm{n} 0}-\frac{\pi}{\alpha}\right)\right)^{2}}\left(\frac{-\cos \frac{\pi}{\alpha}}{\sin ^{4} \frac{\pi}{5}} \times \frac{\alpha}{5}\left(\mathrm{z}-\mathrm{t}_{\mathrm{n} 0}-\frac{\pi}{\alpha}\right)+\frac{1}{\sin ^{3} \frac{\pi}{5}}\right)+\right. \\
& +\frac{\mathrm{f}^{\prime}\left(\mathrm{t}_{\mathrm{n} 0}+\frac{\pi}{\alpha}\right)}{\frac{\alpha}{5}\left(\mathrm{z}-\mathrm{t}_{\mathrm{n} 0}-\frac{\pi}{\alpha}\right)} \times \frac{\mathrm{f}\left(\mathrm{t}_{\mathrm{n} 0}+\frac{2 \pi}{\alpha}\right)}{\frac{\alpha}{5} \sin ^{3} \frac{\pi}{5}}+\frac{-\left(2+3 \cos \frac{2 \pi}{\alpha}\right)}{\left(\frac{\alpha}{5}\left(\mathrm{z}-\mathrm{t}_{\mathrm{n} 0}-\frac{2 \pi}{\alpha}\right)\right)^{2}}\left(\frac{\alpha}{\sin ^{2} \frac{2 \pi}{5} \sin ^{2} \frac{\pi}{5}}\left(\mathrm{z}-\mathrm{t}_{\mathrm{n} 0}-\frac{2 \pi}{\alpha}\right)+\frac{1}{\sin \frac{2 \pi}{5} \sin ^{2} \frac{\pi}{5}}\right)+ \\
& \left.+\frac{\mathrm{f}^{\prime}\left(\mathrm{t}_{\mathrm{n} 0}+\frac{2 \pi}{\alpha}\right)}{\frac{\alpha}{5}\left(\mathrm{z}-\mathrm{t}_{\mathrm{n} 0}-\frac{2 \pi}{\alpha}\right)} \times \frac{1}{\frac{\alpha}{5} \sin \frac{2 \pi}{5} \sin ^{2} \frac{\pi}{5}}\right) \times \sin \frac{\alpha}{5}\left(\mathrm{z}-\mathrm{t}_{\mathrm{n} 0}\right) \times \sin ^{2} \frac{\alpha}{5}\left(\mathrm{z}-\mathrm{t}_{\mathrm{n} 0}-\frac{\pi}{\alpha}\right) \times \sin ^{2} \frac{\alpha}{5}\left(\mathrm{z}-\mathrm{t}_{\mathrm{n} 0}-\frac{2 \pi}{\alpha}\right), \quad(1)
\end{aligned}
$$


where $\mathrm{t}_{\mathrm{n} 0}=\mathrm{n} \frac{5 \pi}{\alpha}, \mathrm{n} \in \mathrm{Z}$, provided that the interpolation series (11) converges uniformly in any bounded region of the complex plane.

Proving Lemma 2, we obtain estimate of the residual of the interpolation series (11), which has the following form

$$
\left|\mathrm{R}_{\mathrm{n}}(\mathrm{z})\right| \leq \mathrm{LG}(\mathrm{z}) \mathrm{C}_{\mathrm{f}} \frac{\alpha}{\alpha-\sigma} \frac{1}{\mathrm{n}},
$$

where $\mathrm{L}$ is a constant,

$$
\begin{gathered}
\mathrm{C}_{\mathrm{f}}=\sup _{\mathrm{t} \in \mathrm{R}}|\mathrm{f}(\mathrm{t})|, \\
\mathrm{G}(\mathrm{z})=\left|\sin \frac{\alpha}{5} \mathrm{z} \times \sin ^{2} \frac{\alpha}{5}\left(\mathrm{z}-\frac{\pi}{\alpha}\right) \times \sin ^{2} \frac{\alpha}{5}\left(\mathrm{z}-\frac{2 \pi}{\alpha}\right)\right|
\end{gathered}
$$

is a function bounded on any bounded region of the complex plane.

\section{Results}

Let's consider a stochastic process $\xi(t), t \in R$ with $M \xi(t)=0$ and covariance function which the representation is

$$
\mathrm{B}(\mathrm{t}, \mathrm{s})=\int_{\Lambda \times \Lambda} \mathrm{f}(\mathrm{t}, \lambda) \overline{\mathrm{f}(\mathrm{s}, \mu)} \mathrm{F}(\mathrm{d} \lambda, \mathrm{d} \mu)
$$

where $\Lambda$ is a set of parameters, $F\left(A_{1}, A_{2}\right)$ is a positive definite additive complex function on $\Lambda \times \Lambda$ such that

$$
\int_{\Lambda \times \Lambda}|\mathrm{F}(\mathrm{d} \lambda, \mathrm{d} \mu)|<+\infty
$$

The function $f(t, \lambda)$ with respect to $t$ is an entire function of exponential type with indicator $\sigma(\lambda)$ such that

$$
\begin{gathered}
\sup _{\lambda \in \Lambda} \sup _{-\infty<\mathrm{t}<+\infty}|\mathrm{f}(\mathrm{t}, \lambda)|=\mathrm{C}_{\mathrm{f}}<+\infty, \\
\sup _{\lambda \in \Lambda} \sigma(\lambda)=\sigma<+\infty .
\end{gathered}
$$

The following theorem holds true.

Theorem 2. Let $\xi(t)$ be a separable stochastic process that satisfies conditions (13)-(16). Then for any $\alpha, \alpha>\sigma$ with probability 1 the following representation holds true

$$
\begin{gathered}
\xi(\mathrm{t})=\sum_{\mathrm{n}=-\infty}^{\infty}\left(\frac{\xi\left(\mathrm{t}_{\mathrm{n} 0}\right)}{\frac{\alpha}{5}\left(\mathrm{t}-\mathrm{t}_{\mathrm{n} 0}\right)} \times \frac{1}{\sin ^{2} \frac{\pi}{5} \sin ^{2} \frac{2 \pi}{5}}+\frac{\xi\left(\mathrm{t}_{\mathrm{n} 0}+\frac{\pi}{\alpha}\right)}{\left(\frac{\alpha}{5}\left(\mathrm{t}-\mathrm{t}_{\mathrm{n} 0}-\frac{\pi}{\alpha}\right)\right)^{2}}\left(\frac{-\cos \frac{\pi}{\alpha}}{\sin ^{4} \frac{\pi}{5}} \times \frac{\alpha}{5}\left(\mathrm{t}-\mathrm{t}_{\mathrm{n} 0}-\frac{\pi}{\alpha}\right)+\frac{1}{\sin ^{3} \frac{\pi}{5}}\right)+\frac{\xi^{\prime}\left(\mathrm{t}_{\mathrm{n} 0}+\frac{\pi}{\alpha}\right)}{\frac{\alpha}{5}\left(\mathrm{t}-\mathrm{t}_{\mathrm{n} 0}-\frac{\pi}{\alpha}\right)} \times\right. \\
\left.\times \frac{1}{\frac{\alpha}{5} \sin ^{3} \frac{\pi}{5}}+\frac{\xi\left(\mathrm{t}_{\mathrm{n} 0}+\frac{2 \pi}{\alpha}\right)}{\left(\frac{\alpha}{5}\left(\mathrm{t}-\mathrm{t}_{\mathrm{n} 0}-\frac{2 \pi}{\alpha}\right)\right)^{2}}\left(\frac{-\left(2+3 \cos \frac{2 \pi}{\alpha}\right)}{\sin ^{2} \frac{2 \pi}{5} \sin ^{2} \frac{\pi}{5}} \times \frac{\alpha}{5}\left(\mathrm{t}-\mathrm{t}_{\mathrm{n} 0}-\frac{2 \pi}{\alpha}\right)+\frac{1}{\sin \frac{2 \pi}{5} \sin ^{2} \frac{\pi}{5}}\right)+\frac{\xi^{\prime}\left(\mathrm{t}_{\mathrm{n} 0}+\frac{2 \pi}{\alpha}\right)}{\frac{\alpha}{5}\left(\mathrm{t}-\mathrm{t}_{\mathrm{n} 0}-\frac{2 \pi}{\alpha}\right)} \times \frac{1}{\frac{\alpha}{5} \sin \frac{2 \pi}{5} \sin ^{2} \frac{\pi}{5}}\right) \times \\
\times \sin \frac{\alpha}{5}\left(\mathrm{t}-\mathrm{t}_{\mathrm{n} 0}\right) \times \sin ^{2} \frac{\alpha}{5}\left(\mathrm{t}-\mathrm{t}_{\mathrm{n} 0}-\frac{\pi}{\alpha}\right) \times \sin ^{2} \frac{\alpha}{5}\left(\mathrm{t}-\mathrm{t}_{\mathrm{n} 0}-\frac{2 \pi}{\alpha}\right) .
\end{gathered}
$$


We obtain that the interpolation series (17) converges with probability 1 to a stochastic process $\xi(t)$ in any bounded domain of changes of parameter $t$.

\section{Discussion}

The research focuses on the modern questions in the theory of stochastic processes. The results in this work are principally new and they are related to the interpolation representations of stochastic processes with non-equidistance interpolation knots. The two schemes of the construction of interpolation knots are investigated, and these schemes are complemented by each other. The work is a continuation and supplement of previously considered problems [10-12]. Obtained results can be applied in the construction of spline-approximation and in the modern theory of information transmission. The further research on this problem is planned in order to obtain new schemes of interpolation representations of stochastic processes with non-equidistance interpolation knots.

\section{Conclusions}

The work is devoted to investigation of interpolation representations of a class of stochastic processes.

The two types of representation knots' groups were constructed. For the first type of knots, the interpolation formula includes the value of the process and its derivatives of the first and second orders. For the second type of knots, the interpolation formula includes the value of the process and its derivatives of first orders. The interpolation formula that uses the value of the process and its derivatives at the knots of interpolation is constructed.

The convergence of the interpolation series to the considered stochastic process with probability 1 has been proved.

\section{References}

[1] Kotel'nikov, V. A. (2006). On the transmission capacity of 'ether' and wire in electric communications. Uspekhi Fizicheskih Nauk, 176 (7), 762-770. doi: 10.3367/ufnr.0176.200607h.0762

[2] Shannon, C. E. (1949). Communication in the Presence of Noise. Proceedings of the IRE, 37 (1), 10-21. doi: 10.1109/jrproc.1949.232969

[3] Shannon, C. E. (1948). Mathematical Theory of Communication. Bell System Technical Journal, 27, 379-423.

[4] Hirurhin, Y. I., Yakovlev, V. P. (1962). Methods of the theory of entire functions in radiophysics, radio and optics theory. Moscow, 220.

[5] Grebenyuk, K. A. (2014). Classical sampling theorem in digital holography. Journal of Physics: Conference Series, 536, 012005. doi: 10.1088/1742-6596/536/1/012005

[6] Farrakhov, R. G., Parfenov, E. V., Gusarov, A. V., Lazarev, D. M., Fatkullin, A. R. (2016). Impedance spectroscopy of the process of hard anodizing of aluminum alloys. Surface Engineering and Applied Electrochemistry, 52 (2), 202-211. doi: 10.3103/s1068375516020058

[7] Kuznetsov, N. A., Sinitsyn, I. N. (2009). Development of Kotel'nikov's sampling theorem. Physics-Uspekhi, 52 (2), 197. doi: 10.3367/UFNe.0179.200902j.0216

[8] Meijering, E. (2002). A chronology of interpolation: from ancient astronomy to modern signal and image processing. Proceedings of the IEEE, 90 (3), 319-342. doi: 10.1109/5.993400

[9] Yaglom, A. M. (1963). Spectral representations for various classes of random functions. Proc. 4-th USSR Math. Congr., 1, 250-273.

[10] Verovkina, G. V. (2013). The interpolation representation of some classes of random processes. Mechanics and Mathematics, 2, 9-11.

[11] Verovkina, G. V., Nagornyi, V. N. (2005). The interpolation representation of one class of random fields. Bulletin of Taras Shevchenko National University of Kyiv Series: Physics \& Mathematics, 1, 31-34.

[12] Verovkina, G. V. (2015). The interpolation representation of some classes of random fields. XVII International Scientific Conference in Honour of Acad. M. Kravchuk, III, 14-16. 